\title{
PERSPECTIVES
}

\section{The use of folded melolabial interpolation flaps to repair full thickness distal nasal defects: A review of technique and results}

\author{
Eamonn Maher ${ }^{*}$, Scott Walen ${ }^{2}$, Ian Maher ${ }^{3}$ \\ ${ }^{1}$ Marshall University School of Medicine, Huntington, West Virginia, USA \\ ${ }^{2}$ Department of Otolaryngology - Head and Neck Surgery, Saint Louis University, St. Louis, Missouri, USA \\ ${ }^{3}$ Department of Dermatology, Saint Louis University, St. Louis, Missouri, USA
}

\begin{abstract}
The nose is a common site for skin cancer and there are several surgical options available for reconstruction after excision. Traditional surgical teaching suggests that in the case of a partial full thickness defect involving the distal nose or ala, a paramedian forehead flap (PMFF) or an intranasal lining flap with free cartilage graft and additional cutaneous flap should be performed. However, each of these options comes with unique disadvantages. The incision and pedicle required for the PMFF can be unsightly and functionally limiting, while the intranasal lining flap is technically challenging and can obstruct the nostril. Herein, we review our experience with a two-stage folded-over melolabial interpolation flap (MLIF) to repair partial full thickness defects of the distal nose and ala in order to recreate the cutaneous covering, nasal rim, and inner mucosal lining.

Keywords: MLIF; melolabial interpolation flap; flap; nasal reconstruction

Citation: Maher E, Walen S, Maher I. The use of folded melolabial interpolation flaps to repair alar full thickness distal nasal defects: A review of technique and results. J Surg Dermatol 2016; 1(3): 140-144; http://dx.doi.org/10.18282/jsd. v1.i3.47.
\end{abstract}

*Correspondence to: Eamonn Maher, Marshall University School of Medicine, Huntington, West Virginia, USA, maher11@marshall.edu.

Received: $22^{\text {nd }}$ February 2016; Accepted: $24^{\text {th }}$ May 2016; Published Online: $23^{\text {rd }}$ August 2016

\section{Introduction}

Every year, there are more new cases of skin cancer reported than cancers of the breast, prostate, lung, and breathing colon combined ${ }^{[1,2]}$. Of these skin cancer cases, a large proportion occur on the nose ${ }^{[3,4]}$. The nose plays an important role with regards to normal breathing - maintaining the patency of the external nasal valve is critical in mitigating airway resistance. In addition, the central and prominent location of the nose on the face renders the presence of any asymmetry as readily noticeable, with adverse effects for the patient in terms of body image and social functions ${ }^{[5,6]}$. Thus, excision of cancers surrounding the nose area present a subsequent challenge in terms of reconstruction and it is crucial that any defects are repaired effectively ${ }^{[7]}$.

The distal nose is composed of thick, sebaceous skin, and fibrofatty tissue overlying a cartilaginous support structure that is present in the tip but not in the ala or soft triangles, as well as an inner mucosal lining. In order to achieve functional and aesthetic results, all layers influenced by the surgical defect must be tended to and replaced. Traditional surgical teaching has held that if a defect involves the lining of the nostril in addition to the cutaneous covering of the nose, it should be repaired in one of two ways: either via a folded paramedian forehead flap $(\mathrm{PMFF})^{[8-11]}$ or an intranasal lining flap with free cartilage graft and additional cutaneous flap ${ }^{[12,13]}$. Repairing the internal lining of the nose or the "mucosa" is imperative in that the mucosa filters, warms, and 
humidifies incoming air. Perhaps the most vital function of the nasal mucosa is the lubrication of the nasal cavity, which prevents nasal crusting and allows a clear passage for air to travel.

The PMFF has the advantage of robust axial-based blood supply via the supratrochlear artery, making it ideal for repairs in areas that have been irradiated or in patient populations with increased risk for reduced distal perfusion like smokers and diabetics ${ }^{[14]}$. Intranasal lining flaps can resurface the nostril lining defects and provide blood supply for the overlying cartilage $\operatorname{graft}^{[12-16]}$. However, each of these procedures comes with its respective disadvantages.

A substantial drawback of the PMFF is the pedicle, which connects the medial eyebrow to the primary defect, interfering with a patient's ability to wear glasses (Figure 1). The vertical donor site scar on the forehead does not conform to the natural cosmetic borders of the face, though it normally heals well. In addition, the PMFF requires some degree of patient isolation and wound maintenance for up to five weeks, which may cause some distress in certain patient populations. Intranasal lining flaps may obstruct the nostril, are technically demanding to perform, and can have tenuous viability in smokers or patients with prior intranasal surgery.

While these options and their inherent drawbacks may be necessary if the lining defect of the wound is large, for wounds with a smaller lining defect a less involved option may be more economical in terms of aesthetic outcome and procedural difficulty.

Herein, we review our experience with a two-stage folded-over cheek interpolation flap to repair distal nasal defects that extend into the nasal rim and intranasal lining. The melolabial interpolated flap (MLIF) carries the advantage of excellent tissue match, robust vascularity, and convenient placement for the donor scar within the melolabial fold (MLF) ${ }^{[17]}$. The flap is designed so that it can be used to resurface the cutaneous covering portion of the nasal defect, with the inferior portion of the flap folded onto itself to resurface the lining defect and to recreate the alar rim. This cheek interpolation flap is a viable option for the reconstruction of distal nasal defects with a partial full thickness component.

\section{Materials and methods}

The first and one of the most important steps in the execution of a MLIF is the design. To begin, a non-stick gauze, foil suture pack, or thermoplastic bandaging material can be used to create a template of the defect ${ }^{[18]}$. Attention to detail during construction of the template is crucial in order to ensure that it accounts precisely for the lining defect, thickness of the alar, rim, and cutaneous covering. The distance from the base of the flap, at the origin of the MLF, to the medial most extent of the primary defect should be measured using a folded gauze. This determines the distance from the superiorly located flap origin that the template should be placed, inferiorly on the cheek. The template is placed just lateral to the MLF, allowing the donor scar to be hidden in the MLF without completely blunting it (Figure 2). As with all other interpolation flaps for nasal repair, the MLIF is rotated medially. Thus, the portion of the template corresponding to the medial portion of the primary defect should point inferiorly on the cheek and the portion of the template corresponding to the lining defect should be oriented laterally. The borders of the template are marked with operative ink, and a standing cutaneous deformity
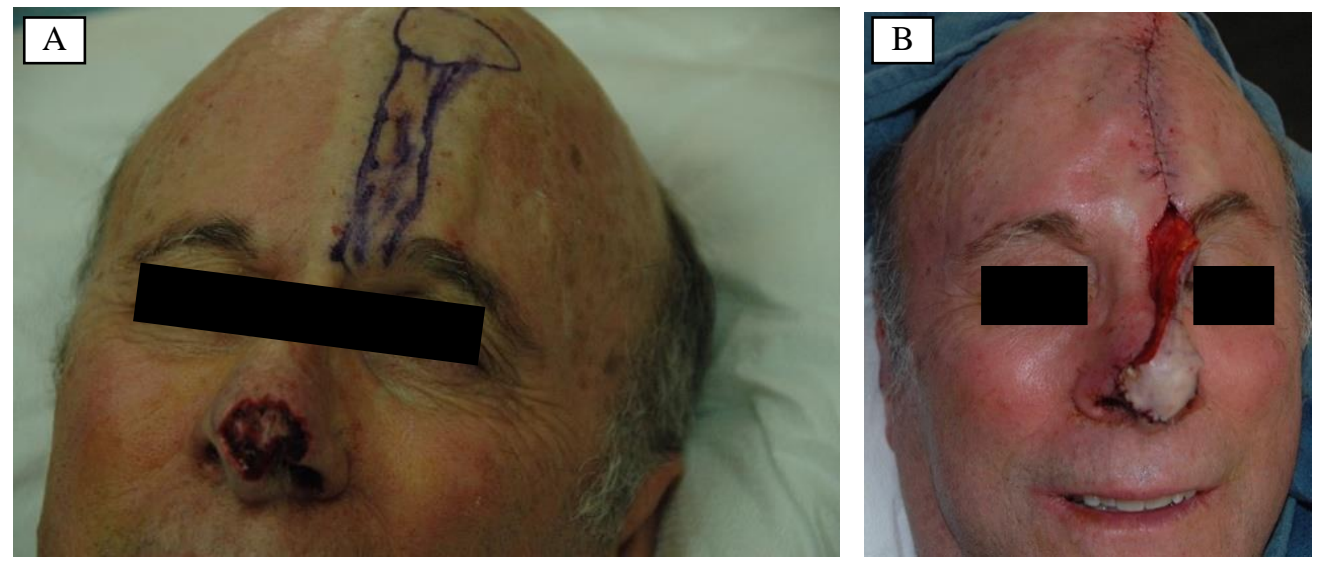

Figure 1. (A) Frontal view of a large nasal tip, soft triangle, and ala defect with design of the PMFF to be used in reconstruction (B) Immediately after creation of PMFF with view of the large pedicle that the procedure requires 

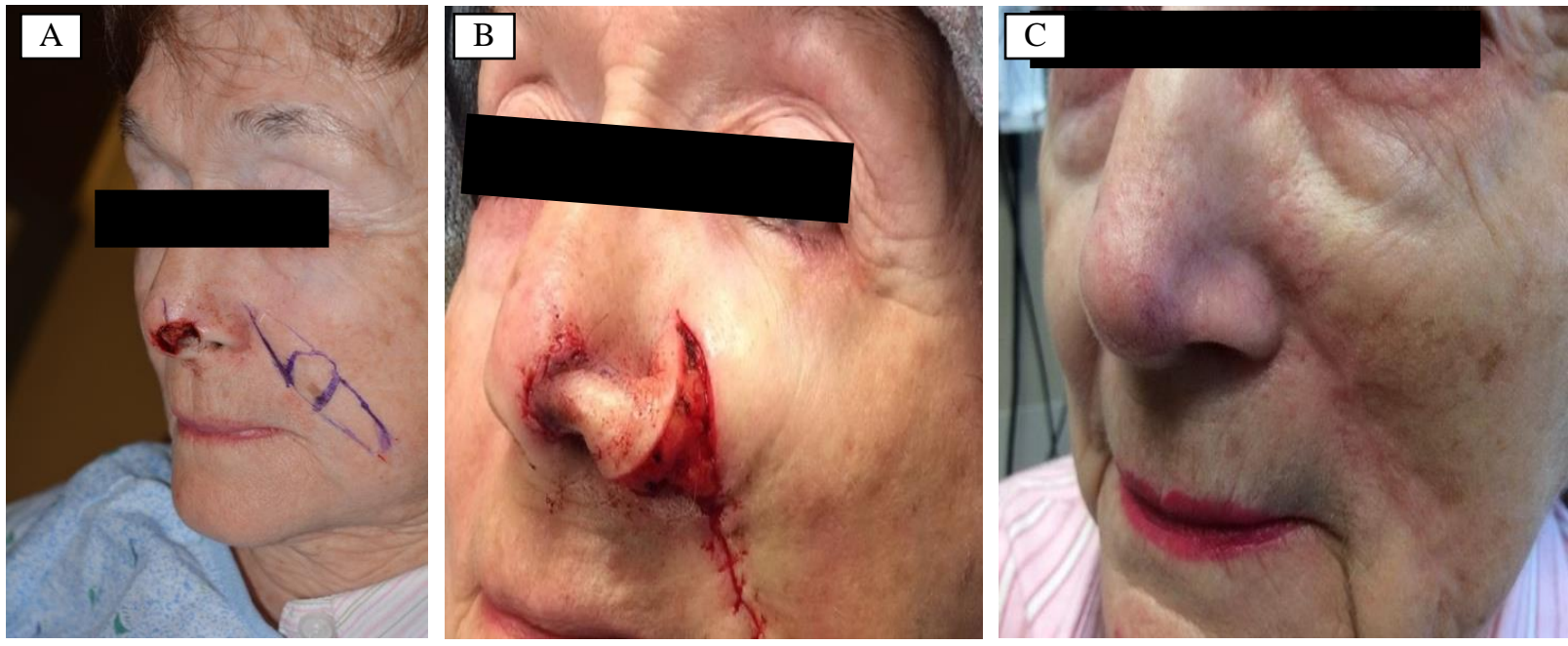

Figure 2. (A) Partial full thickness defect of the ala and soft triangle along with the design for the planned MLIF; (B) Close-up view of the subsequent repair, after creation of the MLIF; (C) Excellent cosmetic results eight weeks post-op

(SCD) is marked distally to the template on the cheek, to aid in donor site closure. Proximally, the flap can be islanded on a musculosubcutaneous pedicle or the skin can be preserved.

Once designing is complete, the primary defect is widely undermined in the submuscular plane. While not necessary for minimizing tension, in our experience this helps minimize post-operative trap door deformity. The flap markings are then incised to the superficial subcutis. The SCD and template portion of the flap should be elevated in the superficial subcutaneous plane. As undermining moves proximally to the template portion of the flap, the undermining plane descends to the supramuscular plane. Undermining should be continued until enough movement is achieved for the template portion of the flap to reach its eventual home in the primary defect with minimal tension. The flap is supplied by perforators from the angular artery at the proximal base of the flap.

After hemostasis is achieved, the cheek donor site is closed in a layered manner. We typically use 4.0 polyglactin 910 and 5.0 poliglecaprone 25 buried vertical mattress sutures, followed by running 5.0 rapidly absorbable gut for the donor site. A buried vertical mattress suture is used to affix the medial portion of the flap to the superior portion of the primary defect by rotating the flap medially into position. The flap is then trimmed and an additional buried vertical mattress is used to affix the flap to the medial portion of the primary defect. The portion of the flap to be infolded, to resurface the alar margin and lining, is fixed with percutaneous simple interrupted sutures. We take a small superficial bite on the flap, with a larger bite on the nasal lining. This asymmetrical suture bite pattern aids with the infolding of the flap. Additional buried vertical mattress sutures are placed at the alar margin before a simple running stitch is used to close the epidermis. The pedicle stalk is wrapped in hemostatic gauze and a pressure bandage is placed. Patients would perform moist wound care on the stalk for three weeks until the second stage, division, and inset procedure.

To begin the second stage of the procedure, the flap pedicle is sectioned, usually at three weeks post-op. The pedicle is sharply divided and the base excised in an elliptical manner, followed by primary closure. The flap on the nose is then inset - an incision is made along the desired alar margin (Figure 3). This incision is continued along the lateral and superior extent of the inset flap, leaving the flap connected to the nose medially. The skin is then sharply undermined off the underlying fibrofatty tissue at the desired thickness, generally $2-3 \mathrm{~mm}$, depending on the thickness of the native nasal skin. Next, undermining in the subnasalis plane is conducted below the flap and in the immediately adjacent tissues.

The fibrofatty tissue which has accumulated beneath the flap should now be easily removed, having been freed from all surrounding tissue. At this point, the flap is carefully trimmed and inset using poliglecaprone buried vertical mattress and running rapidly absorbable gut. To ensure proper healing and maximize aesthetic results, patients generally would be cared for one week and are seen for follow-up at 8-12 weeks post-op.

\section{Results}

The MLIF with fold-over modification was used to repair defects involving cutaneous covering and lining of the nose on 12 patients from July 2013 to December 

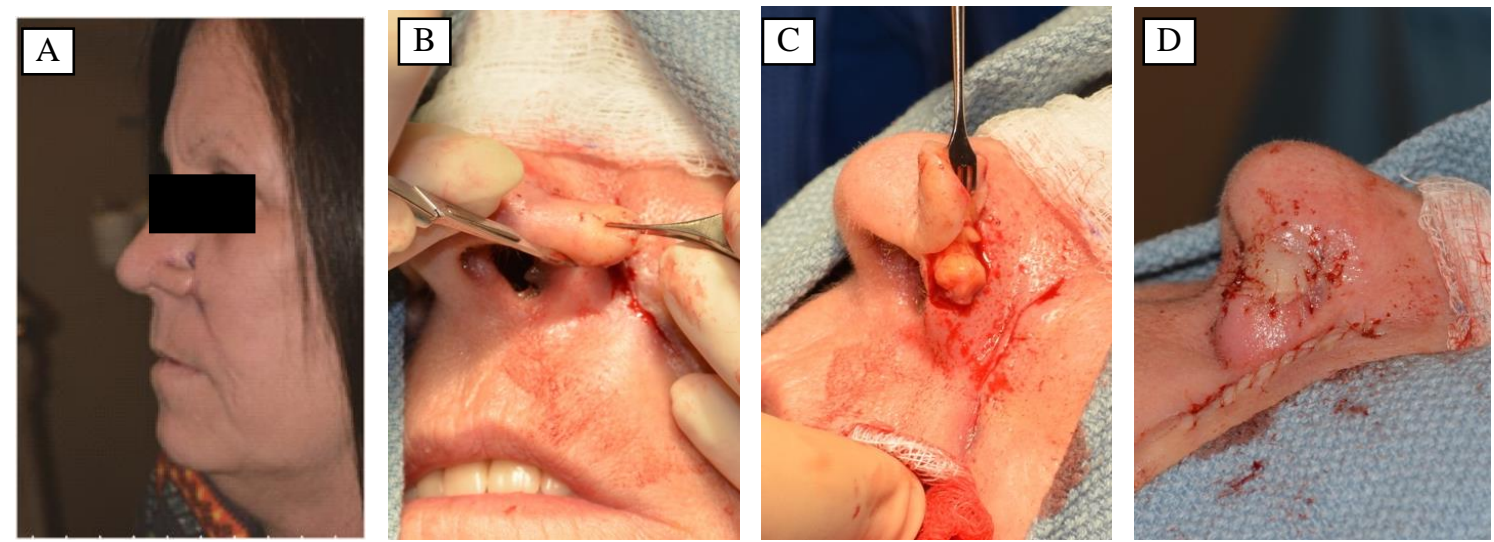

Figure 3. (A) MLIF that has healed well after the first stage of operation, before pedicle division and inset; (B) Initial incision of the MLIF pedicle along the desired alar margin; (C) After division of the MLIF pedicle, during separation of the cutaneous covering from the underlying fibrofatty tissue; (D) Immediately after successful inset of the flap

2015. The average age of the patients was 72 years old and all patients were Caucasian. There were no incidences of hematoma or infection. There was one episode of distal flap sloughing in an active tobacco and methamphetamine abuser. Each patient had at least three months of follow-up from the takedown procedure.

\section{Discussion}

Due to the rich random pattern of vascularity of angular artery perforators, generous donor tissue site, and opportunity for convenient concealment of the incision site within local facial folds, the MLIF has proven to be a reliable option for the reconstruction of distal nasal defects (Figure 4) ${ }^{[19]}$. Traditionally, however, when the defect involves the alar margin and lining of the lower nose, the MLIF has been eschewed in favor of the PMFF or used in conjunction with an intranasal lining flap to repair the lining portion of the defect ${ }^{[8]}$. Both of these methods have potential downsides for patients and
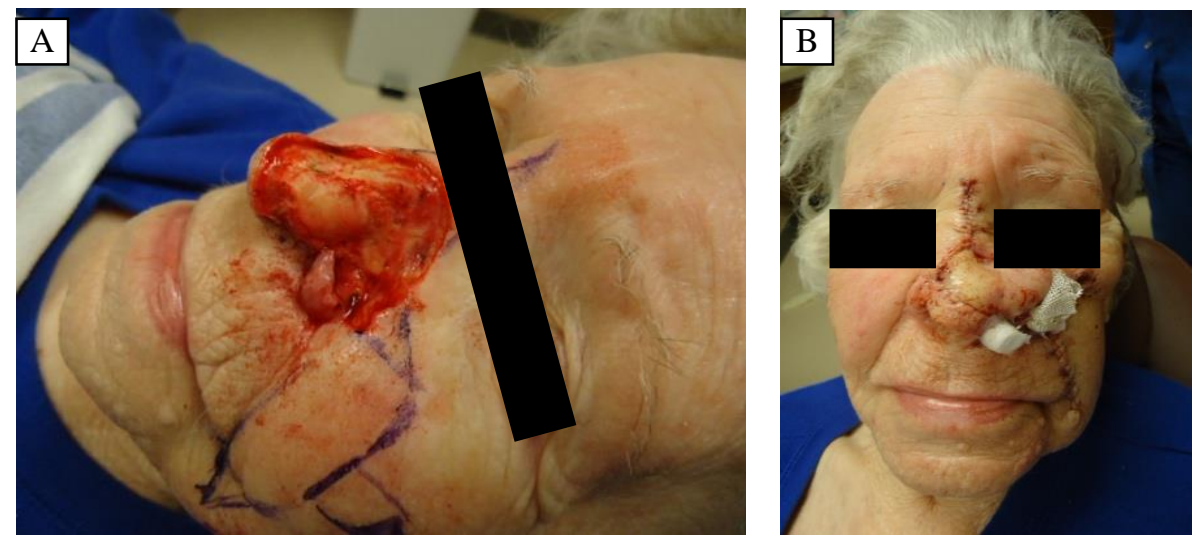

surgeons. Due to the extended incision required and the spatial orientation of the pedicle, the PMFF can interfere with patient's quality of life and daily activities ${ }^{[20]}$. Intranasal lining flaps are technically demanding of the surgeon, and it can result in obstruction of the nasal passageway prior to division, in addition to being dangerous to perform without a secured airway, thus limiting its use in the outpatient setting.

We propose the folded-over MLIF as a viable and relatively technically straightforward tool for repairing distal nasal defects that involve the alar margin and alar lining. The cheek generally provides an excellent color and texture match for distal nasal $\operatorname{skin}^{[6]}$. As long as proper adjustments are made in the template of the wound recipient site, infolding of the flap to resurface the lining component is an efficient option that lacks the morbidity and difficulty of other options for nasal lining. The MLIF - infolded or not - may be a less desirable option for men who have facial hair in the donor region. While flap depilation can be attempted via laser hair removal

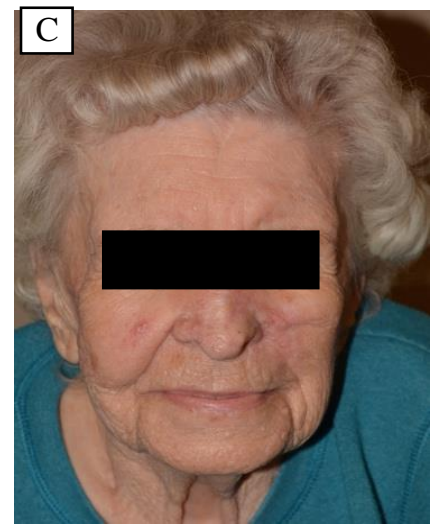

Figure 4. (A) $3.5 \times 3-\mathrm{cm}$ defect involving the left nasal sidewall, ala, alar, rim, and alar lining. The patient would not consent to a PMFF; (B) A MLIF was used for cutaneous covering, and folded on itself to recreate the lining of the ala; (C) Patient with good functional and cosmetic results after a four-month follow-up 
or electrolysis at a later point, the method require repeated treatments that may be inconvenient for patients and is not always effective. Therefore, when possible, it is always best to avoid transferring hair to the nose. Obviously, larger lining defects will require a larger flap to be harvested from the cheek. As such, with increasing size of a defect, the tissue reserve of the cheek site should be assessed for its suitability to be donated without resulting in significant asymmetry. Our experience with employing the MLIF for repairs of complex nasal defects involving both nasal cover and lining has proven it to be a useful and valuable option resulting in a high rate of favorable outcomes. We experienced no flap failures and no complications.

\section{Conclusion}

Reconstruction of the distal nose is a process that has several popular options, including PMFF and intranasal lining flaps accompanied by cartilage grafts and cutaneous flaps. The folded-over MLIF is a viable reconstructive option for partial full thickness defects involving the lining of the distal nose that avoids many of the pitfalls of the PMFF and intranasal lining flaps while still achieving excellent outcomes.

\section{Author contributions}

Maher E spearheaded the composition of the manuscript. Maher I and Walen S had full access to the data and were involved in writing and editing of the manuscript.

\section{Conflict of interest}

The authors declare no potential conflict of interest with respect to the research, authorship, and/or publication of this article.

\section{References}

1. Rogers HW, Weinstock MA, Feldman SR, Coldiron BM. Incidence estimate of nonmelanoma skin cancer (keratinocyte carcinomas) in the US population, 2012. JAMA Dermatol 2015; 151(10): 1081-1086. doi: 10.1001/jamadermatol.2015.1187.

2. Siegel RL, Miller KD, Jemal A. Cancer statistics, 2016. CA Cancer J Clin 2016; 66(1): 7-30. doi: $10.3322 /$ caac. 21332 .

3. Evans GR, Williams JZ, Ainslie NB. Cutaneous nasal malignancies: Is primary reconstruction safe? Head Neck 1997; 19(3): 182-187. doi:10.1002/(SICI)1097-0347 (199705)19:3<182::AID- HED3>3.0.CO;2-Z.

4. Baker SR, Swanson NA. Management of nasal cutaneous malignant neoplasms. An interdisciplinary approach. Arch Otolaryngol 1983; 109(7): 473-479. doi: 10.1001/archoto

\subsubsection{2.}

5. Borah GL, Rankin MK. Appearance is a function of the face. Plast Reconstr Surg 2010; 125(3): 873-878. doi: 10.1097/PRS.0b013e3181cb613d.

6. Rankin M, Borah GL. Perceived functional impact of abnormal facial appearance. Plast Reconstr Surg 2003; 111(7): 2140-2146. doi: 10.1097/01.PRS.0000060105.63 335.0C.

7. Menick FJ. A new modified method for nasal lining: The Menick technique for folded lining. J Surg Oncol 2006; 94(6): 509-514. doi: 10.1002/jso.20488.

8. Menick FJ. A 10-year experience in nasal reconstruction with the three-stage forehead flap. Plast Reconstr Surg 2002; 109(6): 1839-1861. doi: 10.1097/00006534-20020 5000-00010.

9. Singh DJ, Bartlett SP. Aesthetic considerations in nasal reconstruction and the role of modified nasal subunits. Plast Reconstr Surg 2003; 111(2): 639-651. doi: 10.1097/01.PRS.0000041602.85239.93.

10. Yoon T, Benito-Ruiz J, García-Díez E, Serra-Renom JM. Our algorithm for nasal reconstruction. J Plast Reconstr Aesthet Surg 2006; 59(3): 239-247. doi: 10.1016/j.bjps.2005.09.035.

11. Baker SR. Local flaps in facial reconstruction. St. Louis: Mosby Elsevier; 2007. p. 732.

12. Driscoll BP, Baker SR. Reconstruction of nasal alar defects. Arch Facial Plast Surg 2001; 3(2): 91-99.

13. Arden RL, Nawroz-Danish M, Yoo GH, Meleca RJ, Burgio DL. Nasal alar reconstruction: A critical analysis using melolabial island and paramedian forehead flaps. Laryngoscope 1999; 109(3): 376-382. doi: 10.10 97/00005537-199903000-00007.

14. Baker SR, Naficy S. Principles of nasal reconstruction. St. Louis: Mosby; 2002.

15. Burget GC, Menick FJ. Nasal support and lining: The marriage of beauty and blood supply. Plast Reconstr Surg 1989; 84(2): 189-202. doi: 10.1097/00006534-19890800 0-00001.

16. Yellin SA, Nugent A. Melolabial flaps for nasal reconstruction. Facial Plast Surg Clin North Am 2011; 19(1): 123-139. doi: 10.1016/j.fsc.2010.10.010.

17. Maher IA, Garrett AB, Rausch C. Use of thermoplastic bandaging material as a templating medium for the design of interpolation flaps for nasal repair. Dermatol Surg 2012; 38(5): 791-792. doi: 10.1111/j.1524-4725.201 2.02366.x.

18. Fisher GH, Cook JW. The interpolated paranasal flap: A novel and advantageous option for nasal-alar reconstruction. Dermatol Surg 2009; 35(4): 656-661. doi: 10.1111/j.1524-4725.2009.01106.x.

19. Somoano B, Kampp J, Gladstone HB. Accelerated takedown of the paramedian forehead flap at 1 week: Indications, technique, and improving patient quality of life. J Am Acad Dermatol 2011; 65(1): 97-105. doi: 10.1016/j. jaad.2011.01.019.

20. Fader DJ, Baker SR, Johnson TM. The staged cheekto-nose interpolation flap for reconstruction of the nasal alar rim/lobule. J Am Acad Dermatol 1997; 37(4): 614- 619. 